\title{
Regression Analysis of Principal Dimensions and Speed of Aircraft Carriers
}

\author{
J.Y. Bi, Ph. D., \\ Ludong University, China \\ Z. Zong, Ph. D., \\ Dalian University of Technology, China
}

\begin{abstract}
In this paper empirical formulas relating the speed to principal dimensions of aircraft carriers have been obtained through regression analysis of the data of 105 aircraft carriers. To reduce uncertainty as much as possible, aircraft carriers are classified into several different categories. In each category, regression analysis is separately performed such that a variety of regression (empirical) formulas have thus been obtained for possible use at the initial design stage of a carrier. The goodness of fit of these formulas is finally analyzed through variance analysis.
\end{abstract}

Key words: aircraft carriers; speed; principal dimensions; regression; variance

\section{INTRODUCTION}

At the initial design stage of a ship, only speed is provided in the design specifications. Its principal dimensions are usually predicted based on the prescribed ship speed. Or inversely, its speed is predicted based on its principal dimensions. More often than not, the principal dimensions are evaluated either through empirical formulas or through parent ships. This paper is focused on the study of empirical formulas. The method of parent ships will be ignored throughout the paper.

A variety of empirical formulas have been obtained through regression of ship data of various types [2]. For example, there have been empirical formulas for bulk carriers, container ships, oil tankers and passenger liners to estimate their principal dimensions based on given ship speed or vice versa. There is, however, a lack of regression formulas relating the speed to principal dimensions of an aircraft carrier. It is thus needed to find the regression formulas for evaluating the relations between principal dimensions and the speed for aircraft carriers.

To obtain empirical formulas for evaluating speed of an aircraft carrier, 125 sets of data of aircraft carriers [3] are collected. Among them, only 105 have been used in our regression analysis [6] because the information provided in the rest 20 sets of data is not complete. In this paper, regression analysis of available data is presented based on aircraft carriers and regression formulas are obtained for predicting the principal dimensions based on speed performance of an aircraft carrier or vice versa. Different regression formulas are determined through application of various regression forms. The confidence of each regression formula is analyzed through variance [4, 7]. So the most suitable regression formulas for various types of aircraft carriers are given and different regression formulas can be chosen to use for diverse purposes.

\section{REGRESSION ANALYSIS}

The scatters of available data of aircraft carriers are large. To reduce uncertainty aircraft carriers data are divided into different categories. In each category regression analysis is separately performed.

\subsection{Classification by Froude number}

Based on Froude number (Fr) surface vessels of displacement type fall into three categories: low-speed vessel, medium-speed vessel and high-speed vessel. Fr [1] is defined by:

$$
\begin{aligned}
& \text { where: } \quad \mathrm{Fr}=\mathrm{v}_{\mathrm{s}} / \sqrt{\mathrm{gL}} \\
& \mathrm{L}-\text { ship length }[\mathrm{m}], \\
& \mathrm{v}_{\mathrm{s}}-\text { speed }[\mathrm{m} / \mathrm{s}], \\
& \mathrm{g}-\text { acceleration of gravity }\left[\mathrm{m} / \mathrm{s}^{2}\right] .
\end{aligned}
$$

Low-speed ships are those which Froude numbers are smaller than 0.18, that is, $\mathrm{Fr}<0.18$; Medium-speed ships are those which Froude numbers are between 0.18 and 0.30 , that is, $0.18<\mathrm{Fr}<0.30$; High-speed ships are those which Froude numbers are larger than 0.30 , that is, Fr $>0.30$. Analyzing the speed data of aircraft carriers shows that the Froude numbers of aircraft carriers are almost all larger than 0.18 . Therefore the regression analysis will be focused on medium-speed and high-speed aircraft carriers. 


\subsubsection{Medium-speed aircraft carriers}

a) Ayre formula regression

Ayre formula relates Froude number to the ratio of ship length to the cubic root of its displacement. The Ayre formula for civil vessels and merchant ships which lengths are between $120 \mathrm{~m}$ and $140 \mathrm{~m}$ is known to be [5]:

$$
\mathrm{L} / \Delta^{1 / 3}=3.344+10.225 \mathrm{Fr}
$$

where:

$\Delta-$ the displacement [t].

Based on the available data regression analysis is performed for medium-speed aircraft carriers in the form of Ayre formula. The finally obtained regression formula for medium-speed aircraft carriers is:

$$
\mathrm{L} / \Delta^{1 / 3}=12.636 \mathrm{Fr}+4.235
$$

In Fig. 1 the data pairs $\left(\mathrm{L} / \Delta^{1 / 3}\right.$, Fr) based on 49 carriers are plotted. The curve corresponding to the above formula is also given in the figure. The variance estimated from the data is 0.566 , thus showing an unsatisfactory agreement. To improve the accuracy and reduce the variance, quadratic form is employed in the following section for the regression.

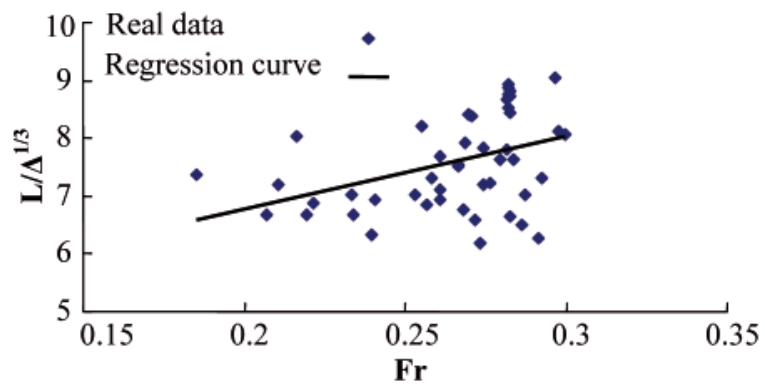

Fig. 1. Ayre regression analysis of medium-speed aircraft carriers

b) Quadratic regression

In the Ayre formula the quantity $\mathrm{L} / \Delta^{1 / 3}$ is assumed a linear function of Froude number, which does not describe the data very well as shown in Fig. 1. A regression formula of quadratic form is therefore used to replace Ayre formula in the hope to reduce the variance. Following the same procedures as above, the regression formula of quadratic form is obtained for medium-speed aircraft carriers as follows:

$$
\mathrm{L} / \Delta^{1 / 3}=202.37 \mathrm{Fr}^{2}-88.407 \mathrm{Fr}+16.663
$$

The data and the regression curve are all plotted in Fig. 2. The variance in this case is 0.541 .

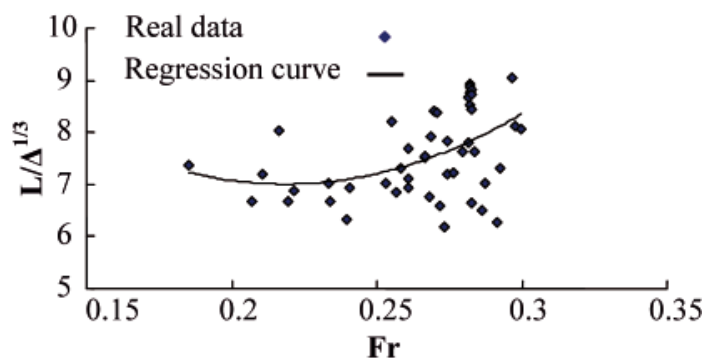

Fig. 2. Polynomial regression analysis of medium-speed aircraft carriers

Comparing the variance of Ayre formula with quadratic formula, we conclude that the latter does not improve much over the former (one being 0.566 and the other 0.541 ). The reason is that the scatters of the data as shown in Figs. 1 and 2 are large. This problem will be discussed further in the following section.

\section{c) Three-dimensional regression}

The above analyses indicate that the regression results are not satisfactory even quadratic form regression is employed. To reduce variance further one more variable is introduced, that is, ship beam into the regression formula. Although the beam is often used to represent stability, the variance is indeed reduced somewhat through introduction of the beam into the regression formula. Because three variables are involved the regression formula is thus a three-dimensional one. The three non-dimensional variables are $\mathrm{L} / \Delta^{1 / 3}, \mathrm{~B} / \Delta^{1 / 3}$ and $\mathrm{Fr}$.

Regression analysis in three-dimensional form has been performed based on 49 sets of data. The obtained threedimensional regression formula is given in the form of:

$$
\begin{aligned}
\mathrm{L} / \Delta^{1 / 3}= & 242.93 \mathrm{Fr}^{2}-9.015\left(\mathrm{~B} / \Delta^{1 / 3}\right)^{2}-70.332 \mathrm{Fr}\left(\mathrm{B} / \Delta^{1 / 3}\right)+ \\
& -46.746 \mathrm{Fr}+38.508\left(\mathrm{~B} / \Delta^{1 / 3}\right)-7.898
\end{aligned}
$$

where:

B - beam $[\mathrm{m}]$.

The data and the regression surface corresponding to the three-dimensional regression formula are plotted in Fig. 3. The variance estimated from the data is 0.483 , having been improved slightly over the quadratic regression formula.

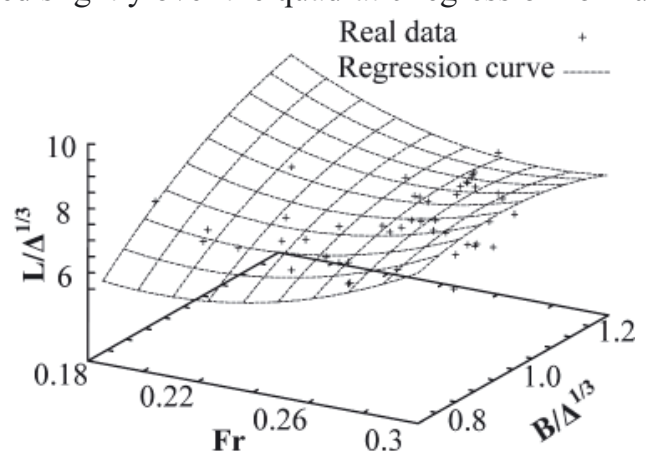

Fig. 3. Three-dimensional regression analysis of medium-speed aircraft carriers

\subsubsection{High-speed aircraft carriers}

a) Ayre formula regression

Again, Ayre formula for high-speed aircraft carriers is first used to represent the linear relationship between displacement length $\mathrm{L} / \Delta^{1 / 3}$ and Froude number (Fr). Regression based on the data of 54 carriers yields the Ayre formula in the form of:

$$
\mathrm{L} / \Delta^{1 / 3}=1.148 \mathrm{Fr}+7.913
$$

The regression curve corresponding to the above formula and the 54 pairs of data are all plotted in Fig. 4 . The variance

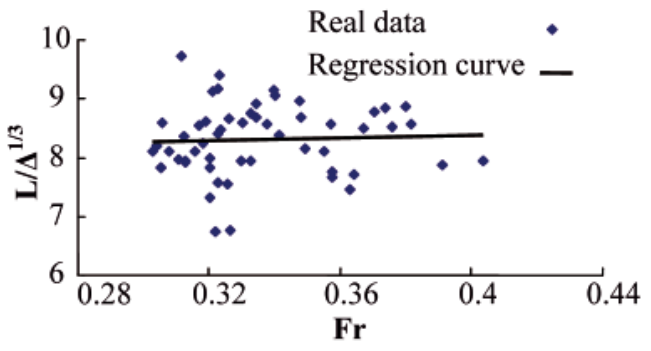

Fig. 4. Ayre regression analysis of high-speed aircraft carriers 
estimated from the data is 0.355 . Comparing with the Ayre formula for medium-speed carriers, we conclude that the Ayre formula for high-speed carriers is better in terms of variance.

\section{b) Quadratic regression}

In order to reduce the variance the quadratic form is used to replace the linear form, that is, quadratic regression formula is obtained to replace the Ayre formula which does not represent the data very well as shown in Fig. 4. The obtained regression formula for high-speed aircraft carriers is:

$$
\mathrm{L} / \Delta^{1 / 3}=-101.58 \mathrm{Fr}^{2}+71.466 \mathrm{Fr}-4.186
$$

The variance in this case is 0.350 by analyzing the data and the regression curve plotted in Fig. 5, showing negligible improvement over the Ayre formula.

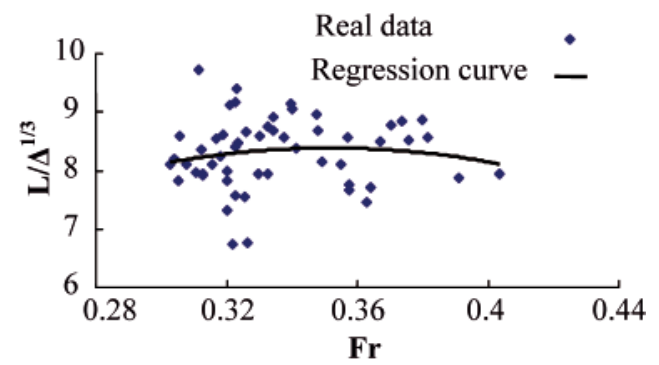

Fig. 5. Polynomial regression analysis of high-speed aircraft carriers

c) Three-dimensional regression

A regression formula of three-dimensional form for highspeed aircraft carriers has been obtained based on the available data of 54 carriers as follows:

$$
\begin{aligned}
\mathrm{L} / \Delta^{1 / 3}= & -98.788 \mathrm{Fr}^{2}+15.538\left(\mathrm{~B} / \Delta^{1 / 3}\right)^{2}-17.314 \mathrm{Fr}\left(\mathrm{B} / \Delta^{1 / 3}\right)+ \\
& +86.640 \mathrm{Fr}-23.222\left(\mathrm{~B} / \Delta^{1 / 3}\right)+3.864
\end{aligned}
$$

The variance in this case is 0.330 . The regression surface is shown in Fig. 6.

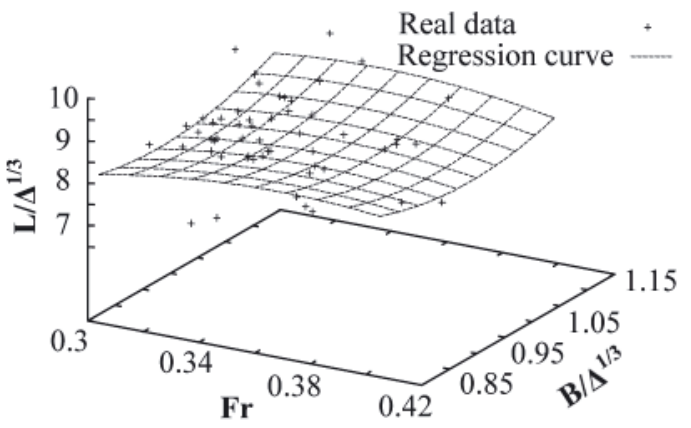

Fig. 6. Three-dimensional regression analysis of high-speed aircraft carriers

\subsection{Classification by Displacement}

Alternatively, aircraft carriers may also fall into three categories: light aircraft carriers, medium aircraft carriers and large aircraft carriers based on displacement. Large aircraft carriers are those which displacements are over 60000 tons, medium aircraft carriers are those which displacements are between 30000 to 60000 tons and light aircraft carriers are those which displacements are less than 30000 tons.

\subsubsection{Light aircraft carriers}

a) Ayre formula regression

Regression analysis for light aircraft carriers in the form of Ayre formula has been performed based on 83 sets of data. The obtained regression formula for light aircraft carriers is:

$$
\mathrm{L} / \Delta^{1 / 3}=10.301 \mathrm{Fr}+4.856
$$

The 83 sets of data and the regression curve are plotted in Fig. 7, and the estimated variance is 0.541 . The variance shows an unsatisfactory agreement. Again, quadratic form is employed in the following section for the regression.

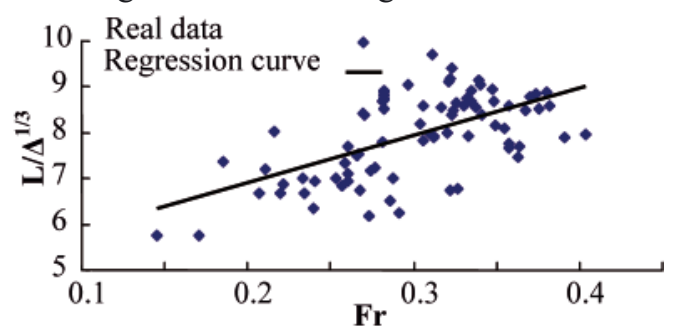

Fig. 7. Ayre regression analysis of light aircraft carriers

b) Quadratic regression

A regression formula of quadratic form for light aircraft carriers is employed in the hope to improve the accuracy. The obtained quadratic regression formula for light aircraft carriers is:

$$
\mathrm{L} / \Delta^{1 / 3}=-54.373 \mathrm{Fr}^{2}+41.579 \mathrm{Fr}+0.513
$$

The variance is estimated to be 0.501 . Again the conclusion is drawn that the quadratic regression does not improve much over the Ayre formula (see Fig. 8).

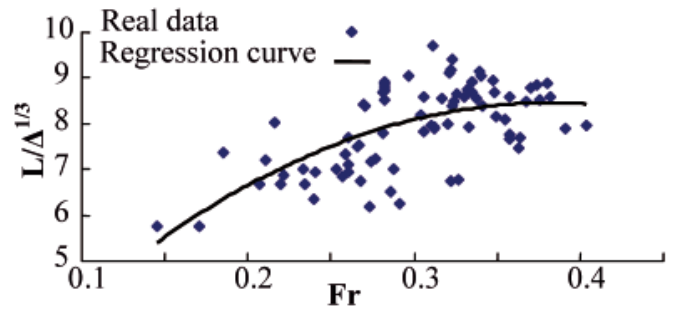

Fig. 8. Polynomial regression analysis of light aircraft carriers

c) Three-dimensional regression

Regression analysis in the form of three-dimensional formula for light aircraft carriers has been performed. The final regression formula in the three-dimensional form is:

$$
\begin{aligned}
\mathrm{L} / \Delta^{1 / 3}=- & 36.888 \mathrm{Fr}^{2}-6.661\left(\mathrm{~B} / \Delta^{1 / 3}\right)^{2}+4.124 \mathrm{Fr}\left(\mathrm{B} / \Delta^{1 / 3}\right)+ \\
+ & 26.755 \mathrm{Fr}+13.540\left(\mathrm{~B} / \Delta^{1 / 3}\right)-4.645
\end{aligned}
$$

The variance estimated from the data is 0.473 . The data and the regression surface are shown in Fig. 9.

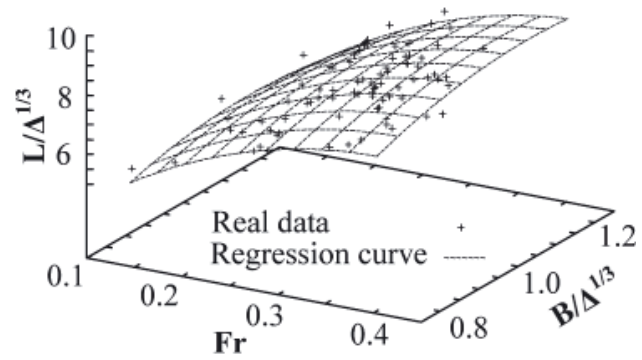

Fig. 9. Three-dimensional regression analysis of light aircraft carriers 


\subsubsection{Medium aircraft carriers}

a) Ayre formula regression

Available data for medium aircraft carriers are not plenty. Only 15 sets of data are used for regression analysis. The obtained regression result in the form of Ayre formula for this case is:

$$
\mathrm{L} / \Delta^{1 / 3}=0.662 \mathrm{Fr}+7.659
$$

The variance estimated from the data is 0.249 . The regression curve is nearly flat in this case as shown in Fig. 10.

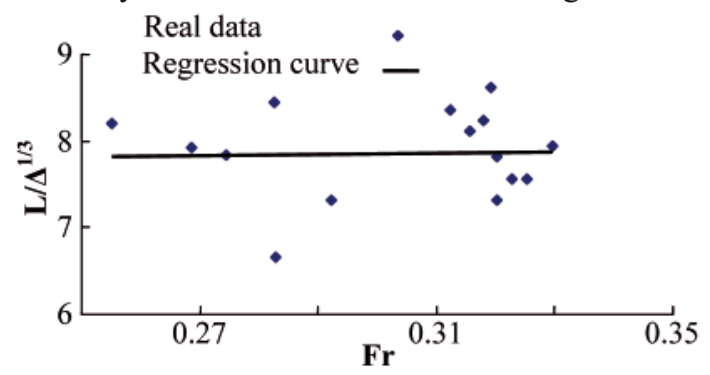

Fig. 10. Ayre regression analysis of medium aircraft carriers

b) Quadratic regression

The regression formula in the form of quadratic form is again used for medium aircraft carriers. The obtained formula is:

$$
\mathrm{L} / \Delta^{1 / 3}=256.08 \mathrm{Fr}^{2}-150.42 \mathrm{Fr}+29.789
$$

The variance estimated from the data is 0.239 . It is just a little better than Ayre formula (see Fig. 11).

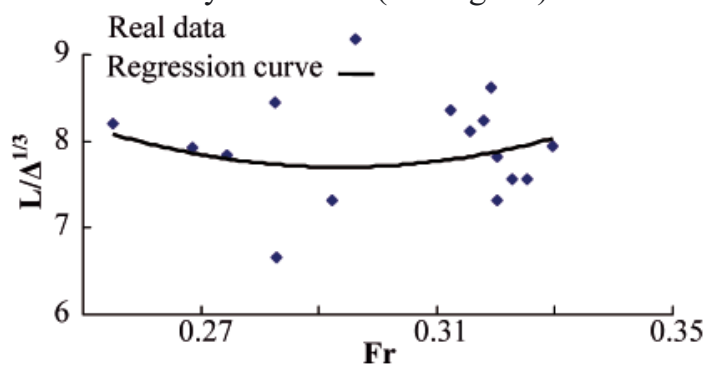

Fig. 11. Polynomial regression analysis of medium aircraft carriers

c) Three-dimensional regression

Based on the above data, regression analysis of threedimensional form is performed for medium aircraft carriers. The following regression formula is obtained:

$$
\begin{gathered}
\mathrm{L} / \Delta^{1 / 3}=209.623 \mathrm{Fr}^{2}-164.361\left(\mathrm{~B} / \Delta^{1 / 3}\right)^{2}+ \\
-209.951 \mathrm{Fr}\left(\mathrm{B} / \Delta^{1 / 3}\right)+81.066 \mathrm{Fr}+ \\
+383.134\left(\mathrm{~B} / \Delta^{1 / 3}\right)-191.213
\end{gathered}
$$

The variance estimated from the data is 0.204 . The regression surface and the data are plotted in Fig. 12.

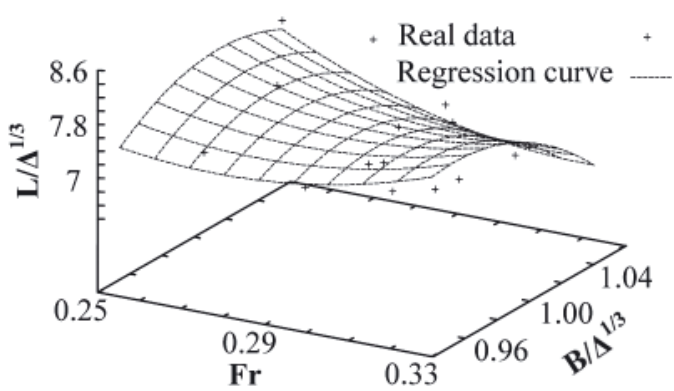

Fig. 12. Three-dimensional regression analysis of medium aircraft carriers

\subsubsection{Large aircraft carriers}

a) Ayre formula regression

Data for large carriers are even less, only 7 sets of data available. Based on the 7 sets of data, the following regression curve is obtained in the form of Ayre formula:

$$
\mathrm{L} / \Delta^{1 / 3}=36.416 \mathrm{Fr}-2.881
$$

The data and regression curve are plotted in Fig. 13. The variance estimated from the data for this case is $5.995 \times 10^{-2}$.

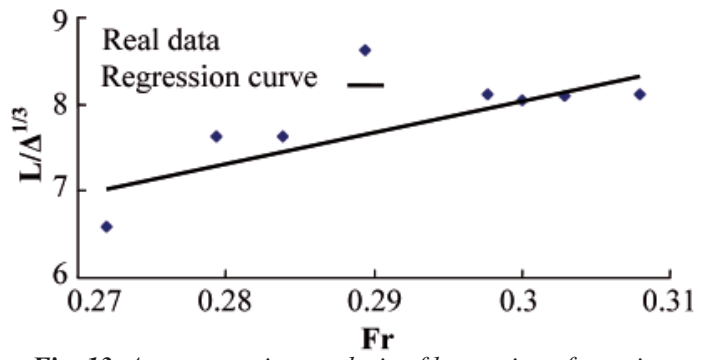

Fig. 13. Ayre regression analysis of large aircraft carriers

b) Quadratic regression

Again quadratic regression analysis is performed for large aircraft carriers. The obtained quadratic regression formula is:

$$
\mathrm{L} / \Delta^{1 / 3}=-1934.4 \mathrm{Fr}^{2}+1158.1 \mathrm{Fr}-165.17
$$

The variance for this case is $1.508 \times 10^{-2}$ (see Fig. 14). Because the sample size is small, both the Ayre formula [(Eq. (12)] and the regression formula of quadratic form [Eq. (13)] are in excellent agreement with the data.

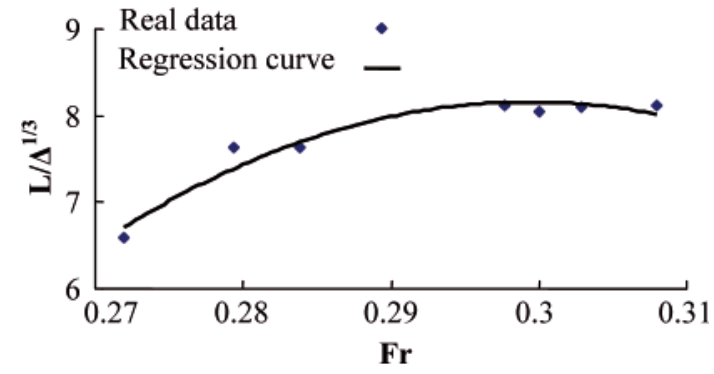

Fig. 14. Polynomial regression analysis of large aircraft carriers

c) Three-dimensional regression

Regression analysis in the form of three-dimensional formula has been performed based on the 7 sets of data. The obtained three-dimensional regression formula is given in the form of:

$$
\begin{aligned}
\mathrm{L} / \Delta^{1 / 3}= & 1848.83 \mathrm{Fr}^{2}+10.308\left(\mathrm{~B} / \Delta^{1 / 3}\right)^{2}-1207.71 \mathrm{Fr}\left(\mathrm{B} / \Delta^{1 / 3}\right) \\
& +95.248 \mathrm{Fr}+338.47\left(\mathrm{~B} / \Delta^{1 / 3}\right)-173.441
\end{aligned}
$$

The data based on 7 carriers and regression surface are plotted in Fig. 15. The variance in this case is $4.561 \times 10^{-3}$.

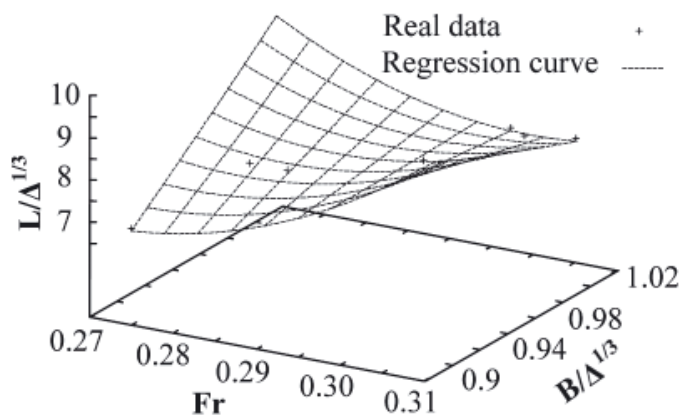

Fig. 15. Three-dimensional regression analysis of large aircraft carriers 


\subsection{Classification by Power}

The third approach used to classify aircraft carriers is based on the power plant. Based on power plant aircraft carriers are classified into conventional-powered aircraft carriers and nuclear-powered aircraft carriers. There are 12 carriers of nuclear-powered class in the world. They are Enterprise, Nimitz class, Improved Nimitz class and Charles de Gaulle. The rest are all conventional-powered.

\subsubsection{Conventional-powered aircraft carriers}

a) Ayre formula regression

The regression formula in the form of Ayre formula for conventional-powered aircraft carriers is:

$$
\mathrm{L} / \Delta^{1 / 3}=10.094 \mathrm{Fr}+4.894
$$

The variance estimated from the data based on 102 carriers is 0.497. The data and regression curve are shown in Fig. 16. In this case, the variance is large due to the large scatters in the data.

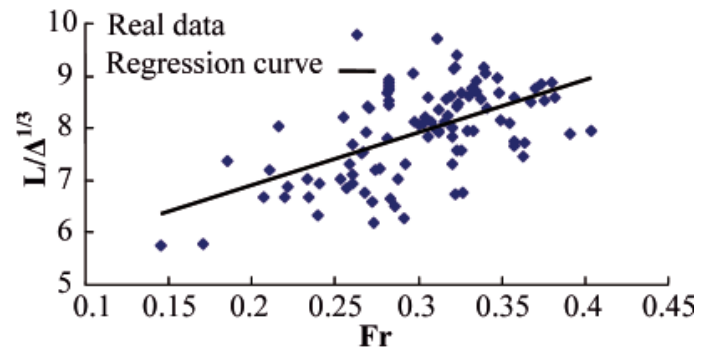

Fig. 16. Ayre regression analysis of conventional-powered aircraft carriers

b) Quadratic regression

The quadratic form regression formula for conventionalpowered carriers based on 102 sets of data is:

$$
\mathrm{L} / \Delta^{1 / 3}=-47.549 \mathrm{Fr}^{2}+37.453 \mathrm{Fr}+1.075
$$

The variance is 0.471 . Both the data and the regression curve are shown in Fig. 17. No much improvement has been observed by replacing Ayre regression formula with the quadratic regression formula.

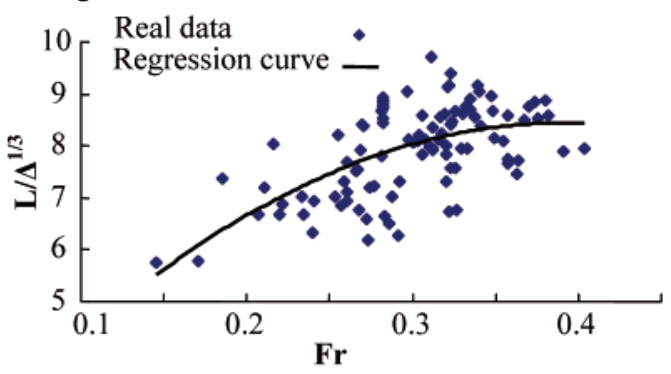

Fig. 17. Polynomial regression analysis of conventional-powered aircraft carriers

c) Three-dimensional regression

Again the following regression formula in three-dimensional form is obtained as follow:

$$
\begin{aligned}
\mathrm{L} / \Delta^{1 / 3}= & -24.546 \mathrm{Fr}^{2}-4.816\left(\mathrm{~B} / \Delta^{1 / 3}\right)^{2}-3.430 \mathrm{Fr}\left(\mathrm{B} / \Delta^{1 / 3}\right)+ \\
& +26.435 \mathrm{Fr}+12.236\left(\mathrm{~B} / \Delta^{1 / 3}\right)-4.026
\end{aligned}
$$

The variance estimated from the data based on 102 carriers is 0.448 . The correlation between the data and regression surface is clearly shown in Fig. 18.

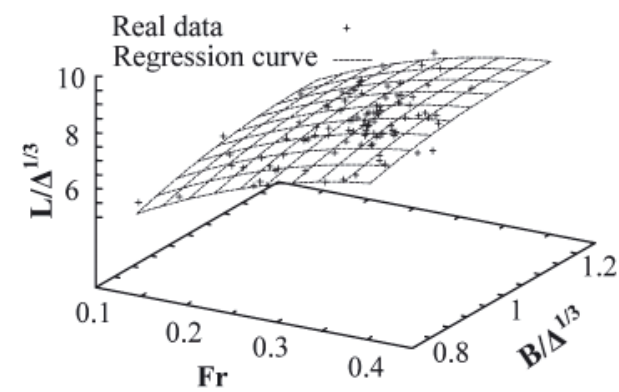

Fig. 18. Three-dimensional regression analysis of conventional-powered aircraft carriers

\subsubsection{Nuclear-powered aircraft carriers}

a) Ayre formula regression

There are 12 nuclear-powered aircraft carriers ever built. They are however pertaining to only four different types, that is, Enterprise (only one), Nimitz class (3), Improved Nimitz class (7) and Charles de Gaulle (only one). Therefore, there have only been four sets of data available for nuclear-powered carriers. The regression formula relating the displacement length to Froude number is:

$$
\mathrm{L} / \Delta^{1 / 3}=28.769 \mathrm{Fr}-0.277
$$

The variance estimated from the data is $3.049 \times 10^{-2}$ (see Fig. 19).

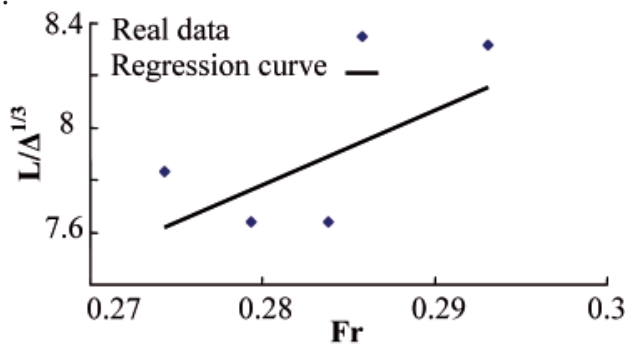

Fig. 19. Ayre regression analysis of nuclear-powered aircraft carriers

b) Quadratic regression

A regression formula in the quadratic form has also been tried with excellent results obtained due to the small sample size. The final regression formula is:

$$
\mathrm{L} / \Delta^{1 / 3}=4904.8 \mathrm{Fr}^{2}-2757.6 \mathrm{Fr}+395.21
$$

The variance in this case is $9.943 \times 10^{-5}$. In Fig. 20, the data and the regression formula are in perfect agreement as expected because there are only four sets of data.

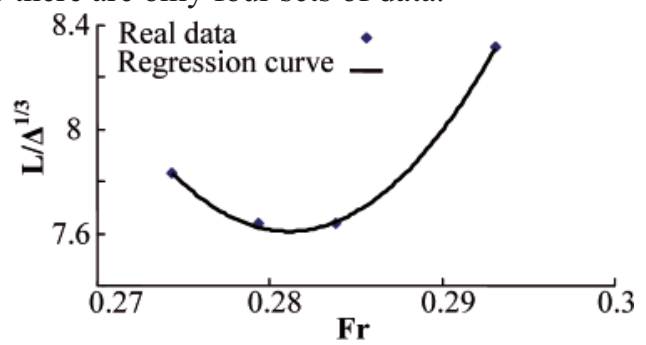

Fig. 20. Polynomial regression analysis of nuclear-powered aircraft carriers

c) Three-dimensional regression

The regression formula in three-dimensional form has also been performed with excellent results. The obtained threedimensional regression formula is: 


$$
\begin{aligned}
\mathrm{L} / \Delta^{1 / 3} & =789.71 \mathrm{Fr}^{2}-411.993\left(\mathrm{~B} / \Delta^{1 / 3}\right)^{2}-637.36 \mathrm{Fr}\left(\mathrm{B} / \Delta^{1 / 3}\right)+ \\
& +154.037 \mathrm{Fr}+992.573\left(\mathrm{~B} / \Delta^{1 / 3}\right)-498.617
\end{aligned}
$$

The variance in this case is $4.896 \times 10^{-10}$. It is a perfect surface as shown in Fig. 21.

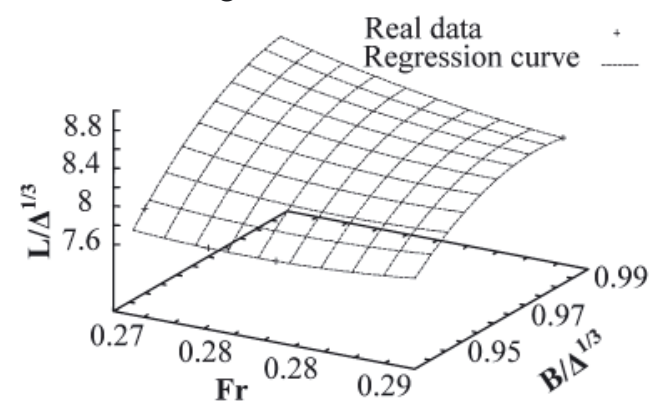

Fig. 21. Three-dimensional regression analysis of nuclear-powered aircraft carriers

\section{DISCUSSION}

Regression theory states that the smaller the variance is the better the regression is. For comparison all the previous results are placed in Tab. 1. From the Table, conclusion may be drawn that in general the variances of regression formulas of quadratic form are smaller than those of the Ayre formula, thus the latter being better than the former. Moreover, the variances of threedimensional regression formulas are smaller than the other two forms of regression formulas. Therefore, three-dimensional regression formulas are the best among the three regression methods. Although ship beam B is generally not present in such regression formulas for civil vessels, three-dimensional regression formulas for carriers are recommended because they do improve the regression accuracy apparently.

Of particular interest are large carriers and nuclear-powered carriers. Their regression is excellent. This may be attributed to the fact that large carriers are smaller in number and thus the sample scatters are small, too. Another reason is that these large carriers are built following almost the same plans. Take nuclear-powered carriers for instance. Twelve nuclear-powered carriers are in fact built based on four different categories.

\section{EXAMPLE}

In this section, several examples are given to demonstrate the use of the regression formulas obtained in this paper. Based on given displacement, length and beam of a given aircraft carrier, its speed is predicted as shown in Table 2. In the Table, the real-ship speed is also given for comparison.

In general, 3-D regression formulas are better than the other. From Table 2, however, there are occasional cases where speed predictions from quadratic form or Ayre form are better than 3-D form, but the differences are not significant. Therefore, three-dimensional regression formulas are recommended for use at the initial design stage of a carrier.

\section{CONCLUSION}

Empirical formulas relating speed with other principal dimensions of a ship play an important role at the initial stage of design. In this paper such empirical formulas for carriers are

Tab. 1. Variance analysis for each category of carriers

\begin{tabular}{|c|c|c|c|c|}
\hline \multirow{2}{*}{ Classification } & \multirow{2}{*}{ Type of aircraft carriers } & \multicolumn{3}{|c|}{ Principal dimensions regression } \\
\cline { 3 - 5 } & Medium-speed & $\begin{array}{c}\text { Ayre } \\
\text { regression }\end{array}$ & $\begin{array}{c}\text { Quadratic } \\
\text { regression }\end{array}$ & $\begin{array}{c}\text { Three-dimensional } \\
\text { regression }\end{array}$ \\
\hline \multirow{3}{*}{ Speed } & 0.566 & 0.541 & 0.483 \\
\hline \multirow{3}{*}{ Pisplacement } & High-speed & 0.355 & 0.350 & 0.330 \\
\cline { 2 - 5 } & Light & 0.541 & 0.501 & 0.473 \\
\cline { 2 - 5 } & Medium & 0.249 & 0.239 & 0.204 \\
\hline & Large & $5.995 \times 10^{-2}$ & $1.508 \times 10^{-2}$ & $4.561 \times 10^{-3}$ \\
\cline { 2 - 5 } & Conventional-power & 0.497 & 0.471 & 0.448 \\
\hline & Nuclear-power & $3.049 \times 10^{-2}$ & $9.943 \times 10^{-5}$ & $4.896 \times 10^{-10}$ \\
\hline
\end{tabular}

\begin{tabular}{|c|c|c|c|c|c|c|c|c|}
\hline \multirow[b]{2}{*}{ Name } & \multirow[b]{2}{*}{$\begin{array}{c}\text { Displacement } \\
{[\mathbf{t}]}\end{array}$} & \multirow[b]{2}{*}{$\begin{array}{c}\text { Length } \\
{[\mathrm{m}]}\end{array}$} & \multirow[b]{2}{*}{$\begin{array}{l}\text { Beam } \\
{[\mathrm{m}]}\end{array}$} & \multirow[b]{2}{*}{$\begin{array}{l}\text { Real } \\
\text { speed } \\
{[\mathrm{m} / \mathrm{s}]}\end{array}$} & \multirow[b]{2}{*}{ Formula to use } & \multicolumn{3}{|c|}{ speed predicted } \\
\hline & & & & & & $\begin{array}{c}\text { 3-D } \\
\text { formula } \\
{[\mathrm{kn}]}\end{array}$ & $\begin{array}{c}\text { quadratic } \\
\text { formula } \\
\text { [kn] }\end{array}$ & $\begin{array}{c}\text { Ayre } \\
\text { formula } \\
{[\mathrm{kn}]}\end{array}$ \\
\hline Theodore Roosevelt & 82500.0 & 332.5 & 40.9 & 31.0 & nuclear powered & 31.0 & 30.9 & 30.5 \\
\hline Theodore Roosevelt & 82500.0 & 332.5 & 40.9 & 31.0 & large & 30.5 & 31.4 & 32.1 \\
\hline John F. Kennedy & 61850.0 & 320.6 & 39.4 & 33.0 & $\begin{array}{c}\text { conventional } \\
\text { powered }\end{array}$ & 33.0 & 33.7 & 34.7 \\
\hline John F. Kennedy & 61850.0 & 320.6 & 39.4 & 33.0 & Large & 33.5 & 33.2 & 32.9 \\
\hline Lexington & 39450.0 & 270.5 & 32.1 & 33.0 & $\begin{array}{l}\text { conventional } \\
\text { powered }\end{array}$ & 29.4 & 29.1 & 30.3 \\
\hline Lexington & 39450.0 & 270.5 & 32.1 & 33.0 & medium & 31.9 & 32.5 & 43.3 \\
\hline
\end{tabular}

Tab. 2. The comparison of estimated speed and real speed 
studied and obtained through regression analysis of 105 sets of data. These data are classified into different categories based on speed, displacement and power plant. In each category three forms of regression formulas are obtained for speed prediction or for prediction of principal dimensions. The variances of these formulas are also given for correct choice of the formula used in the design.

\section{Acknowledgements}

The authors are grateful to the financial support from Chinese NSF (51221961), Programme 973 (2010CB832704, 2013CB036101), High-tech Ship programme and Chinese NSF (51279030).

\section{BIBLIOGRAPHY}

1. Carrica P.M., Wilson R.V. and Stern F.: Unsteady RANS simulation of the ship forward speed diffraction problem, Computers \& Fluids, 35, 6, 545-570, 2006.

2. Gu M.T.: The principles of ship design, Shanghai Jiao Tong University Press, Shanghai, 2001.

3. Ireland B.: The Illustrated Guide to Aircraft Carriers of the World, Hermes House, London, 2005.
4. Paroka D., Ohkura Y. and Umeda N.: Analytical Prediction of Capsizing Probability of a Ship in Beam Wind and Waves, Journal of Ship Research, 50, 2, 187-195(9) , 2006.

5. Schneekluth H. and Bertram V.: Ship Design for Efficiency and Economy, Butterworth \& Heinemann, Oxford, 1998.

6. Sen A. and Srivastava M.: Regression Analysis: Theory, Methods, and Applications, Springer, New York, 1997.

7. Zong Z:: Information-Theoretic Methods for Estimating Complicated Probability Distributions, Elsevie Science, Amsterdam, 2006.

\section{CONTACT WITH THE AUTHORS}

J.Y. Bi, Ph. D., School of Transportation, Ludong University, Yantai 264025, China e-mail: bijunying@gmail.com

Z. Zong, Ph. D., School of Naval Architecture Engineering, Dalian University of Technology, Dalian 116024, China 